\title{
A comparative study of the technical feasibility of complete salpingectomy versus tubal ligation as a method of tubal sterilisation during caesarean delivery
}

\author{
Taru Gupta, Divya Baruhee*, Sunaina Agarwal
}

Department of Obstetrics and Gynecology, Obstetrics and Gynaecology, ESIC PGIMSR, Basaidarapur, New Delhi, India

Received: 15 October 2021

Revised: 14 November 2021

Accepted: 15 November 2021

*Correspondence:

Dr. Divya Baruhee,

E-mail: divyabaruhee765@gmail.com

Copyright: (C) the author(s), publisher and licensee Medip Academy. This is an open-access article distributed under the terms of the Creative Commons Attribution Non-Commercial License, which permits unrestricted non-commercial use, distribution, and reproduction in any medium, provided the original work is properly cited.

\section{ABSTRACT}

Background: Recent theory on ovarian malignancy suggests its origin from primary foci in fallopian tubes. Prophylactic salpingectomy may benefit in risk reduction of ovarian cancer. The aim of the study was to compare the feasibility of complete salpingectomy with bilateral tubal ligation was made during caesarean delivery in women desirous of permanent sterilisation.

Methods: We conducted a prospective interventional randomized control study in department of obstetrics and gynaecology, ESI-PGIMSR Basaidarapur, New Delhi from October 2018 to February 2021. 70 women participated in this study desiring permanent sterilisation during caesarean delivery. Patients were randomized into two groups who either underwent bilateral tubal ligation [group A $(\mathrm{N}=35)$ ] or complete bilateral salpingectomy [group $\mathrm{B}(\mathrm{N}=35)$ ].

Results: Procedure was completed in $91 \%$ patients in group A and $77 \%$ in group B $(\mathrm{p}>0.05)$ making both the procedures feasible. Total operative time (min) in group A and B were 47 and 55 respectively $(\mathrm{p}<0.01)$. Mean sterilisation procedure time (min) in group A was 5 and group B was $6(\mathrm{p}=0.13)$. No significant difference was seen in intra-operative blood loss (400 ml in group A vs $300 \mathrm{ml}$ in group B), percentage decrease in post-op haemoglobin $(\mathrm{g} / \mathrm{dl})(4.56 \pm 2.96 \mathrm{in}$ group A vs 3.91 \pm 2.7 in group B), post-operative serum Anti-mullerian hormone (AMH) levels (ng/ml) (group A$0.965 \pm 0.12$ vs $0.7 \pm 0.09$ in group B) in the two groups.

Conclusions: Salpingectomy as an alternative to tubal ligation should be discussed with suitable patients desirous of permanent sterilisation during caesarean delivery.

Keywords: Salpingectomy, Ovarian cancer, Tubal ligation, Sterilisation

\section{INTRODUCTION}

Ovarian malignancy is one of the most fatal gynaecological cancer. It ranks $7^{\text {th }}$ in prevalence and is the $8^{\text {th }}$ leading cause of cancer related deaths in women worldwide. $^{1}$

Approximately $90 \%$ of the ovarian malignancies are of epithelial origin of which high grade serous epithelial cancers follow an aggressive course and present in advanced stage. ${ }^{2}$ Majority of ovarian malignancies, in particular high grade serous epithelial subtype, have been shown to originate from a primary-foci within the fallopian tubes. $^{3}$

During ovulation, there is a breach of the ovarian surface which transfers the oocyte to the fimbria. The fimbriae closely adhere to the ovaries at the site of extrusion of the oocyte from the ovarian surface. During this process, there is a transfer of the tubal epithelial cells to the ovarian cortex. Implantation of these cells on the raw surface of the ovaries result in the formation of an inclusion cyst which 
may undergo a process of malignant transformation. ${ }^{4}$ Therefore, the focus has now shifted towards the primary prevention of the disease. Eliminating the primary foci of malignancy by performing a prophylactic salpingectomy, may reduce the risk of ovarian cancer.

Tubal sterilisation during caesarean section, in women desiring permanent sterilisation provides an opportunity to discuss the benefits of salpingectomy. American College of Obstetrics and Gynaecology recommends that potential benefits of salpingectomy should be discussed with patients as an alternative method of tubal sterilisation. ${ }^{5}$

The aim of the study was to assess the feasibility of salpingectomy as a method of tubal sterilisation during caesarean delivery in our set up.

\section{METHODS}

A prospective, interventional single-blinded comparative randomized control study was conducted in the department of obstetrics and gynaecology, ESI PGIMSR Basaidarapur, New Delhi. Ethical clearance was taken from the Institution Ethical Committee.

Inclusion criteria were women of 25-45 years of age, with period of gestation (POG) $>35$ weeks, having 2 healthy living children with younger child 2 years or above and no known Gross congenital anomaly (GCA) in the present pregnancy, desiring permanent sterilisation during caesarean delivery.

Patients with known medical disorder (diabetes mellitus, hypertension, heart disease, bleeding disorder), on immunosuppressive therapy or history of tubal surgery were excluded from the study. After explaining the risks and benefits of the procedure, 70 patients were enrolled in the study. Written informed consent was taken from both wife and husband.

The study population was randomized into two groups. Group A $(\mathrm{N}=35)$ patients underwent tubal sterilisation by modified Pomeroy's technique and group B $(\mathrm{N}=35)$ patients underwent complete bilateral salpingectomy. Randomisation was done by a computer-generated randomization table.

In group $\mathrm{A}$, double ligation of the midportion of the tube was done by no 1-0 chromic catgut followed by transection of the tube (Figure 1A). In complete salpingectomy, the fimbriae were separated the ovary, ovarian vessels are identified and isolated. An avascular window was made in the mesosalpinx at a distance of $10 \mathrm{~mm}$ from the cornua. 2 clamps were applied and the fallopian tube were divided and suture ligated with 2-0 chromic catgut. $10 \mathrm{~mm}$ of the tube on the cornua was left behind (Figure 1B).

If salpingectomy could not be completed then the standard tubal ligation by modified Pomeroy's technique was attempted.

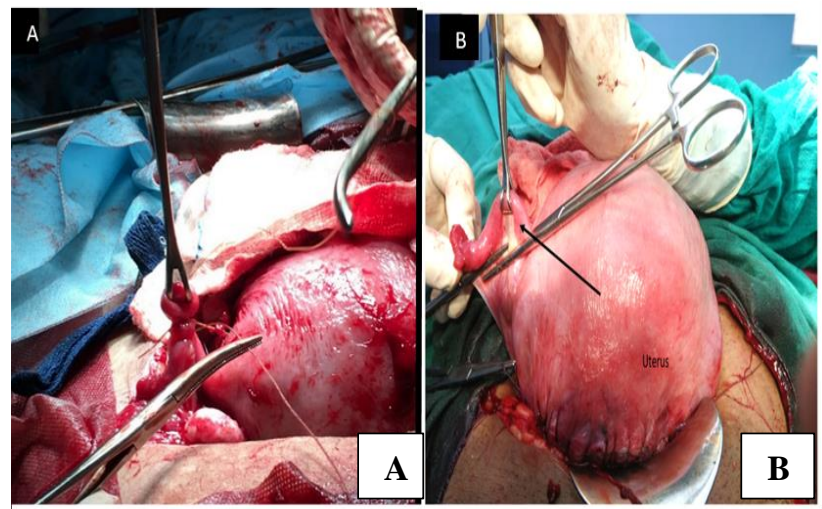

Figure 1: Tubal ligation performed by modified (A) Pomeroy's method; and (B) salpingectomy.

The primary objective of this study was to observe if complete salpingectomy could be done bilaterally. The procedure was termed technically feasible if more than $50 \%$ of the cases could be completed. Secondary objectives of this study were to compare the total operative time (from skin incision to skin closure), time taken for the tubal sterilisation procedure, clinically estimated blood loss, change in hemoglobin from preoperative period to postoperative day 3, surgical complications intraoperatively and in the postoperative period between two groups. Serum Anti-mullerian hormone (AMH) was measured preoperatively and at 6 weeks postpartum. The total time taken for procedure was recorded by a stopwatch used in operation theatre. Blood loss was estimated by measuring blood in the suction apparatus, clot size and the gauze visual analogue. ${ }^{6}$

Clinical estimation of the blood loss was also done. Patients were followed up to 6 weeks postpartum for any postoperative complications like surgical site infection, postpartum fever/sepsis, need for Blood transfusion (BT), post-operative ileus, need for readmissions, or relaparotomy. Statistical analysis was done using SPSS 21.0. Quantitative variables were compared using the independent t-test/Mann Whitney test. Qualitative variables were compared using the Chi-square test/Fischer's exact test. A p value of $<0.05$ was considered statistically significant.

\section{RESULTS}

The median Interquartile range (IQR) age in years in group A was 30 (28-32) and in group B was 32 (29-34). Mean gestational age (in weeks) in group $\mathrm{A}$ and $\mathrm{B}$ were $38.52 \pm 1.22$ and $38.16 \pm 1.19$ respectively with no significant difference between them.

Sterilisation was completed in $91.43 \%$ in group $\mathrm{A}(\mathrm{N}=32)$ and $77.14 \%$ in $\mathrm{B}(\mathrm{N}=27)$ with no significant difference between the them $(\mathrm{p}>0.05)$. Reason for non-completion in group A were postpartum hemorrhage (66.67\%; $\mathrm{N}=2$ ), (adhesions $33.33 \% ; \mathrm{N}=1$ ). Reason for non-completion in group $\mathrm{B}$ were postpartum haemorrhage $(50 \% ; \mathrm{N}=2)$, 
adhesions (37.50\%; $\mathrm{N}=3)$; placenta accreta $(\mathrm{N}=1)$, diagnosed intraoperatively for which obstetric hysterectomy was performed. Tubal sterilisation was successfully completed in more than $50 \%$ of patients in both groups $(\mathrm{N}=32$ in group $\mathrm{A}, \mathrm{N}=27$ in group $\mathrm{B})$, thus, confirming the technical feasibility of complete salpingectomy as a method of sterilisation. The mean total operative time ( $\mathrm{min})$ in group B $(55.26 \pm 5.35)$ was greater than in group A $(47.94 \pm 5.52)$ and was statistically significant ( $\mathrm{p}$ value $<0.0001$ ) (Table 1). The sterilisation procedure time (min) in group A was 5 (4-6) and group B was 6 (5-6) with no significant difference between them (p value $=0.13$ ) (Figure 2). The mean pre-operative haemoglobin $(\mathrm{g} / \mathrm{dl})$ in group $\mathrm{A}$ and $\mathrm{B}$ were $11.3 \pm 1.2$ and $11.55 \pm 1.21$ respectively. Haemoglobin $(\mathrm{g} / \mathrm{dl})$ at day 3 in group $\mathrm{A}$ and $\mathrm{B}$ were 10.76 \pm 1.14 and 11.04 \pm 1.17 (p value $=0.13$ ). The percentage decrease in hemoglobin $(\mathrm{g} / \mathrm{dl})$ in group A was $4.56 \pm 2.96$ and group B was 3.91 \pm 2.7
( $\mathrm{p}$ value $=0.38)$ (Figure 3$)$. The median (IQR) blood loss (ml) in group A was $400(300-450)$ and B was 350 (300450) $(\mathrm{p}$ value $=0.54)($ Figure 4$)$.

No significant difference was seen in the distribution of post-operative complications like post-operative ileus, need for blood transfusion, ICU admission, anaesthetic complications, iatrogenic injury, post-partum fever/sepsis, wound infection, and relaparotomy between group A and $B$ ( $p$ value $>0.05)$.

A rise in post-operative serum $\mathrm{AMH}$ at 6 weeks was seen in both groups. Serum AMH (ng/ml) in pre-op patients was $0.91 \pm 0.79$ and in post-op patients was $0.96 \pm 0.68$ in group A $(\mathrm{N}=32)(\mathrm{p}=0.48)$. Serum $\mathrm{AMH}$ in preoperative patients was $0.60 \pm 0.50$ and in post-op patients was $0.70 \pm 0.51$ in group $\mathrm{B}(\mathrm{N}=27)(\mathrm{p}=0.48)$ (Figure 5 and 6$)$.

Table 1: Comparison of total operative time (min) between group $A$ and $B$.

\begin{tabular}{|c|c|c|c|c|c|}
\hline Total operative time & $A(\mathbf{N}=32)$ & $\mathbf{B}$ & Total & P value & Test performed \\
\hline Mean \pm SD & $47.94 \pm 5.52$ & $55.26 \pm 5.35$ & $51.6 \pm 6.53$ & \multirow{3}{*}{$<0.0001$} & \multirow{3}{*}{ t-test; 5.63} \\
\hline Median (IQR) & $46(44-52)$ & $54(50.5-59.5)$ & $52(46.5-56)$ & & \\
\hline Range & $38-62$ & $48-65$ & $38-65$ & & \\
\hline
\end{tabular}

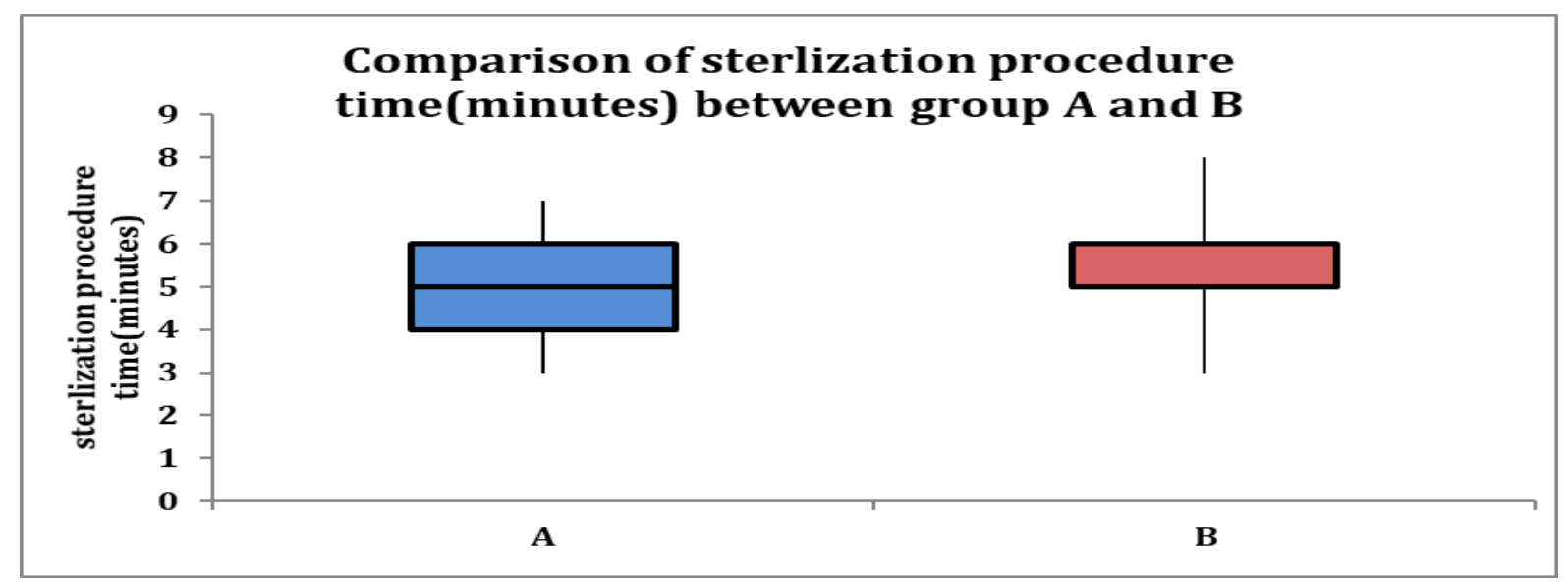

Figure 2: Comparison of sterilisation procedure time $(\mathrm{min})$ between group A and B (non-parametric variable, boxWhisker plot).

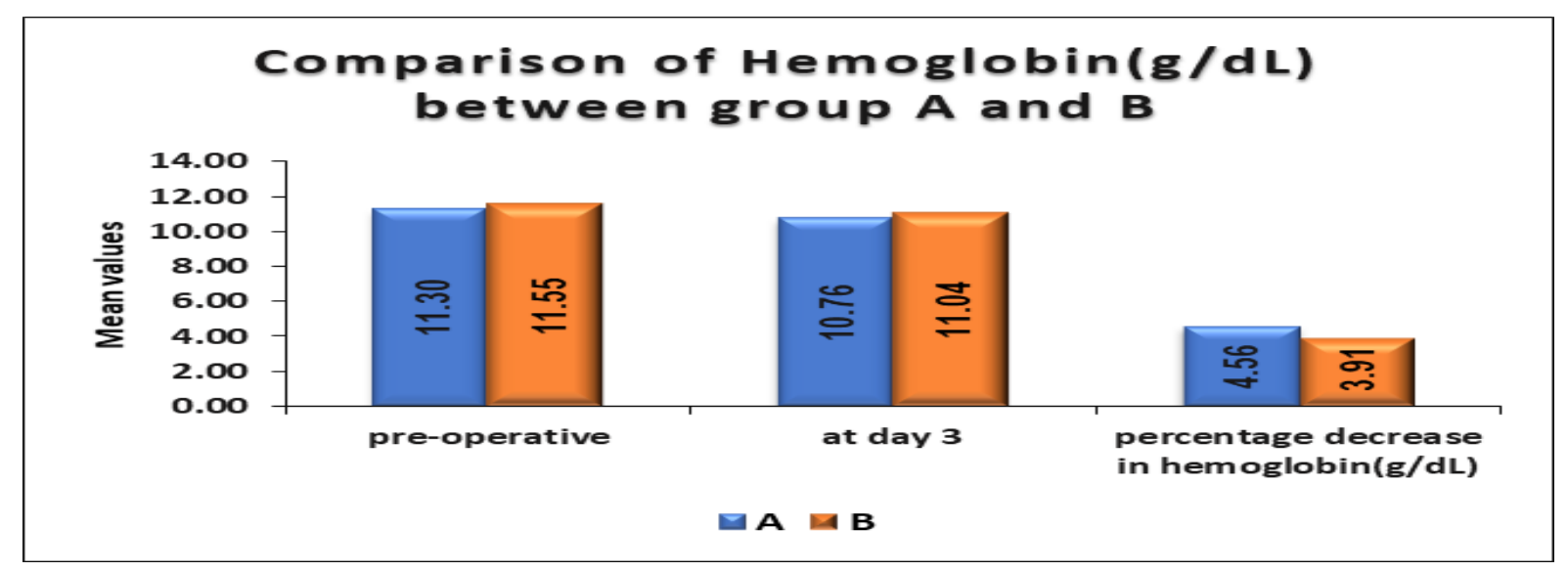

Figure 3: Comparison of hemoglobin (g/dl) between group A and B (parametric variables). 


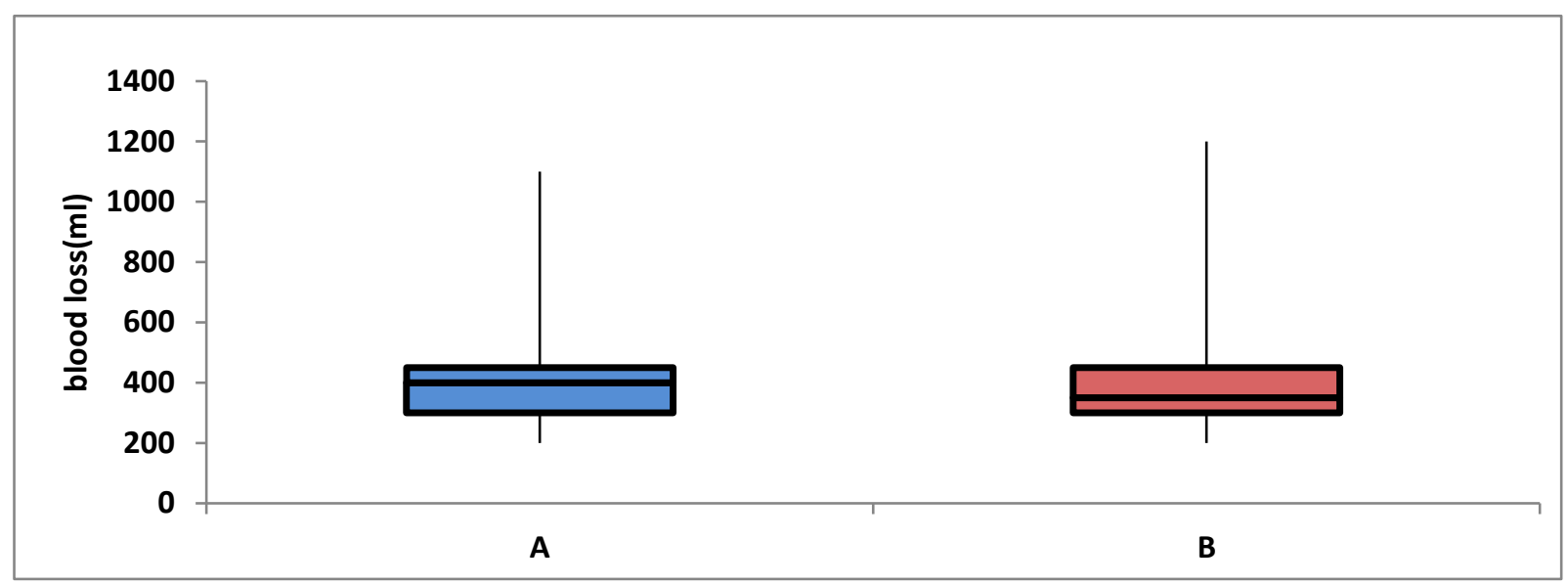

Figure 4: Comparison of blood loss ( $\mathrm{ml})$ between group A and B (non-parametric variable, box-Whisker plot).

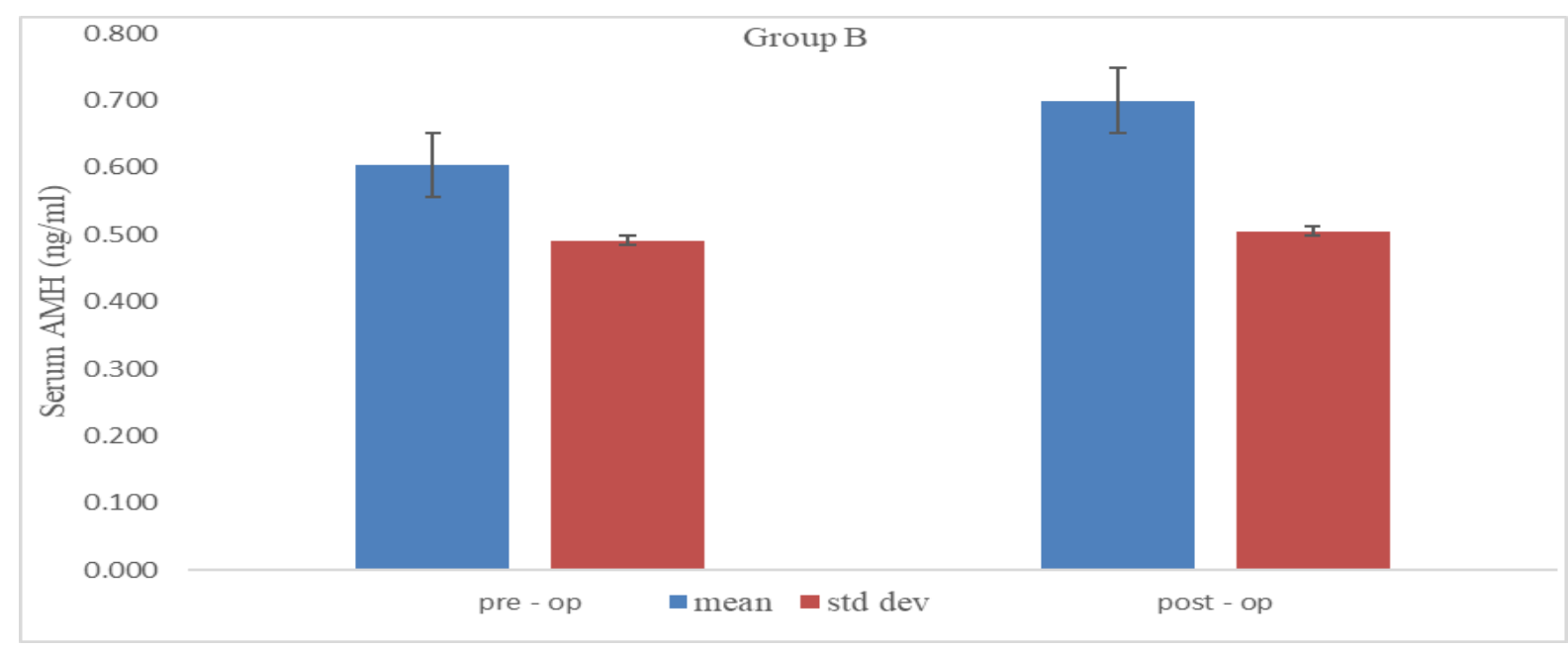

Figure 5: Comparison of pre-op and post-op serum AMH (ng/ml) between the two groups- graph representing the relation between pre-op and post-op serum $\mathrm{AMH}$ level in tubal ligation group.

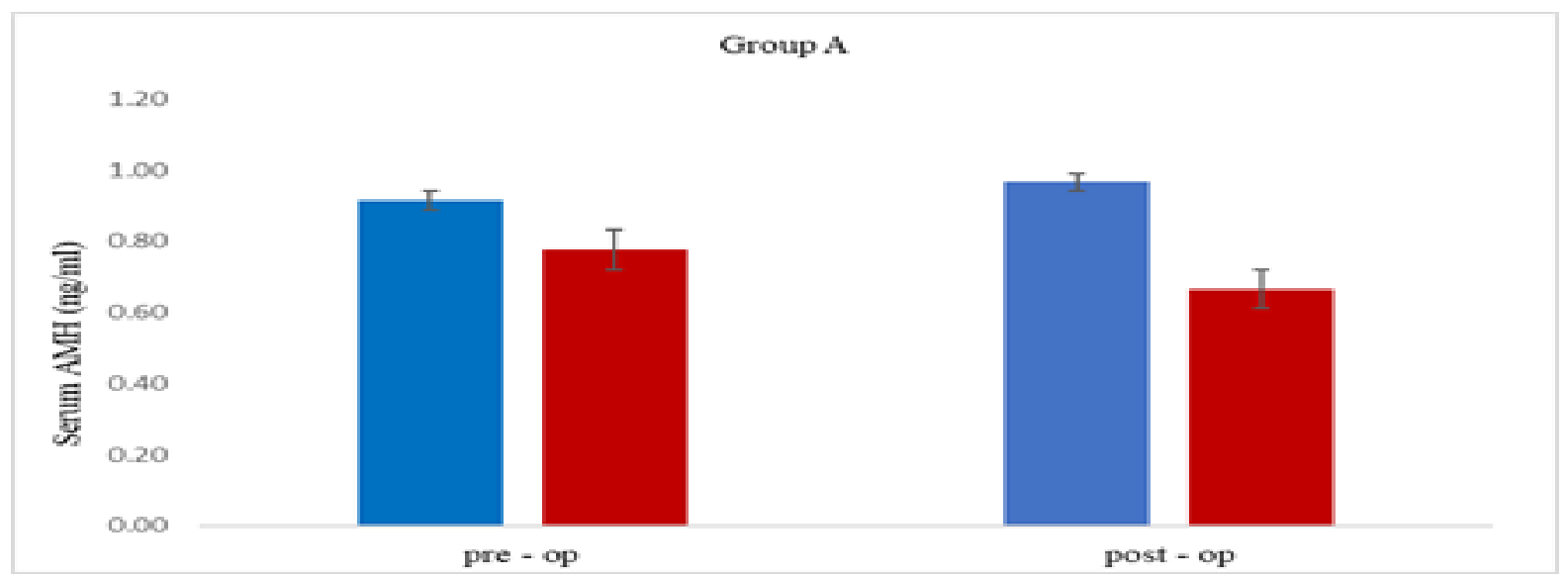

Figure 6: Comparison of pre-op and post-op serum AMH (ng/ml) between the two groups- graph representing relation between pre-op and post-op serum $\mathrm{AMH}$ level in salpingectomy group.

\section{DISCUSSION}

In the study, primary objective was to compare the technical feasibility of complete salpingectomy vs partial salpingectomy (standard bilateral tubal ligation). The two groups had comparable variables like age, body mass index, period of gestation, number of previous LSCS, and associated comorbidities. The sterilisation procedure was successfully completed in $91.43 \%$ cases in A and $77.14 \%$ in group $B(p=0.18)$. Sterilisation procedures in both 
groups could be successfully completed in more than $50 \%$ of patients in the study and were thereby termed as technically feasible. No significant difference was seen in the distribution of reason for non-completion of sterilisation procedure between groups $\mathrm{A}$ and $\mathrm{B}(\mathrm{p}=0.78)$.

Subramanyam et al compared the technical feasibility of successful completion of bilateral salpingectomy with bilateral tubal ligation. Salpingectomy was completed in $68 \%$ of the cases while bilateral tubal ligation was completed in $95 \%$ of the cases ( $\mathrm{p}$ value $=0.002) .{ }^{7}$ A similar finding was observed in our study.

Danis et al compared the difference between postpartum salpingectomy and postpartum Bilateral tubal ligation (BLTL) in terms of duration of the procedure and complication rate. In their study, postpartum sterilisation was performed within 24 hours of vaginal delivery. The average surgical time was $59.13 \mathrm{~min}( \pm 16 \mathrm{~min})$ in the BTL cohort and $71.44 \mathrm{~min}( \pm 5.81 \mathrm{~min})$ in the salpingectomy group $(\mathrm{p}$ value $=0.003) .{ }^{8}$ In the present study, significant difference was seen in total operative time between group $\mathrm{A}$ and B ( $\mathrm{p}$ value $<0.0001)$, however no significant difference was seen in sterilisation procedure time between groups ( $\mathrm{p}$ value $=0.13)$. The increased total operative time in salpingectomy group could be because of the extra time taken to enter the abdominal cavity due to adhesions as a result of previous surgeries. There were more patients in group B with previous LSCS compared to group A (25 vs 20) thereby leading to an increased total operative time in group B than group A.

Shinar et al compared the complication rates of bilateral total salpingectomy vs partial salpingectomy during caesarean delivery. The mean total operative time was comparable for partial and total salpingectomy (median time was $35 \mathrm{~min}$ in both groups, $\mathrm{p}$ value $=0.92) .{ }^{9}$ No significant difference was seen in the rate of complications like post-partum fever, wound infection, hospitalisation length, estimated blood loss, and need for blood transfusions within one month postpartum. Our observation is consistent in terms of total blood loss and complication rates with the study conducted by Shinar et al.

Parikh et al retrospectively evaluated the safety of salpingectomy vs tubal ligation by observing the preoperative change in the haemoglobin during caesarean delivery and post vaginal delivery. They observed no significant decrease in $\mathrm{Hb}$ in either group. The mean difference in pre and post-op $\mathrm{Hb}$ was $0.18 \mathrm{mg} / \mathrm{dl}$ (p value $=0.99)$. They observed a mean decrease of $0.04 \mathrm{mg} / \mathrm{d}$ in the salpingectomy group compared to the tubal ligation group. ${ }^{10}$

In the present study, no significant difference was seen in hemoglobin $(\mathrm{g} / \mathrm{dl})$ pre-operative, and at day 3 between groups ( $\mathrm{p}$ value>0.05). The percentage decrease in haemoglobin $(\mathrm{g} / \mathrm{dl})$ in $\mathrm{A}$ was $4.56 \pm 2.96$ and $\mathrm{B}$ was 3.91 \pm 2.7 . The possible explanation for less fall in $\mathrm{Hb}$ in group B could be because there were more patients with previous LSCS in group B and the healed fibrous tissue led to less bleeding and less fall in haemoglobin.

Ganer Herman et al compared short term ovarian reserve in salpingectomy vs tubal ligation during caesarean deliveries in patients willing for permanent sterilisation. Blood samples were obtained for serum AMH preoperatively and at 6-8 weeks postpartum and there was no significant different between the groups, with an average increase in serum AMH of $0.58 \pm 0.98$ vs $0.39 \pm 0.41 \mathrm{ng} / \mathrm{ml}$ in the salpingectomy and tubal ligation groups, respectively $(\mathrm{p}=0.45) .{ }^{11}$ In the present study, the mean serum AMH in the post-operative ligation group was $0.96 \mathrm{ng} / \mathrm{ml}$ and the salpingectomy group was $0.70 \mathrm{ng} / \mathrm{ml}$. The average increase in the ligation group was $0.05 \mathrm{ng} / \mathrm{ml}$ and in salpingectomy group was of $0.10 \mathrm{ng} / \mathrm{ml}$ (p value $=0.48$ ). There was no decline in serum $\mathrm{AMH}$ in either of the groups. The possible explanation could be that the sterilisation procedures did not cause any significant reduction in the blood supply of the ovaries, as it has rich collateral supply from branches of the ovarian and uterine artery.

Since we did not know the pre-pregnancy baseline serum $\mathrm{AMH}$, we cannot infer from our data that there was an actual increase in serum AMH post-sterilisation procedure. This was a drawback of the study and further studies should be directed comparing the pre-pregnancy values of serum AMH with the post sterilisation values.

Although the sample size was small ( $\mathrm{N}=35$ in each group), the study was adequately powered for the primary outcome.

Limitations of our study was the lack of knowledge of the outcome of complete salpingectomy in comparison to bilateral tubal ligation on ovarian cancer risk reduction for which a very long follow up period is needed $(\sim 30-40$ years). Epidemiological population-based studies are the only source of all data regarding the ovarian cancer risk reduction with the procedure.

Also, the study should have been conducted on a larger cohort of patients, but as it is a relatively uncommon procedure for sterilisation in our population acceptance may still take time by both clinicians and patients. In rare circumstances, patients post salpingectomy may want to conceive later in life, and recanalization is not an option in such patients. However, all merits and demerits of the procedure were explained to all the patients preoperatively with written informed consent and salpingectomy was conducted in patients with at least 2 healthy living children.

To the best of our knowledge, only a few studies have been conducted internationally so far comparing the technical feasibility of complete salpingectomy with partial salpingectomy at the time of caesarean delivery. 
Salpingectomy may be a novel cost-effective method of ovarian malignancy risk reduction.

\section{CONCLUSION}

The study concluded that complete salpingectomy as a method of tubal sterilisation is a technically feasible and safe procedure. However, the total operative time for the salpingectomy group was more than the ligation group. Blood loss and fall in haemoglobin was comparable in both groups. There were no major complications present in either group. Considering the proven benefits of salpingectomy in the prevention of ovarian malignancy, the novel option of salpingectomy should be discussed with patients as a method of permanent tubal sterilisation explaining the merits and demerits of the procedure.

Funding: No funding sources

Conflict of interest: None declared

Ethical approval: The study was approved by the Institutional Ethics Committee

\section{REFERENCES}

1. Ferlay J, Soerjomataram I, Dikshit R, Eser S, Mathers $\mathrm{C}$, Rebelo M, et al. Cancer incidence and mortality worldwide: sources, methods and major patterns in GLOBOCAN 2012. Int J Cancer. 2015;136(5):35986.

2. Desai A. Epithelial ovarian cancer: An overview. World J Transl Med. 2014;3(1):1.

3. Berek JS, Kehoe ST, Kumar L, Friedlander M. Cancer of the ovary, fallopian tube, and peritoneum. Int $\mathbf{J}$ Gynaecol Obstet. 2018;143(2):59-78.

4. Kurman RJ, Shih IM. The origin and pathogenesis of epithelial ovarian cancer: a proposed unifying theory. Am J Surg Pathol. 2010;34(3):433-43.
5. ACOG Committee Opinion No. 774: Opportunistic Salpingectomy as a Strategy for Epithelial Ovarian Cancer Prevention. Obstet Gynecol. 2019;133(4):279-84.

6. Algadiem E, Aleisa AA, Alsubaie HI, Buhlaiqah NR, Algadeeb JB, Alsneini HA. Blood Loss Estimation Using Gauze Visual Analogue. Trauma Mon. 2016;21(2):34131.

7. Subramaniam A, Blanchard CT, Erickson BK, Szychowski J, Leath CA, Biggio JR, et al. Feasibility of Complete Salpingectomy Compared With Standard Postpartum Tubal Ligation at Cesarean Delivery: A Randomized Controlled Trial. Obstet Gynecol. 2018;132(1):20-7.

8. Danis RB, Della BCR, Richard SD. Postpartum Permanent Sterilization: Could Bilateral Salpingectomy Replace Bilateral Tubal Ligation? J Minim Invasive Gynecol. 2016;23(6):928-32.

9. Shinar S, Blecher Y, Alpern S, Many A, Ashwal E, Amikam U, Cohen A. Total bilateral salpingectomy versus partial bilateral salpingectomy for permanent sterilization during cesarean delivery. Arch Gynecol Obstet. 2017;295(5):1185-9.

10. Parikh P, Kim S, Hathcock M, Torbenson VE, Raju R. Safety of salpingectomy at time of delivery. J Matern Fetal Neonatal Med. 2021;34(17):2765-70.

11. Herman H, Gluck O, Keidar R, Kerner R, Kovo M, Levran D, et al. Ovarian reserve following cesarean section with salpingectomy vs tubal ligation: a randomized trial. Am J Obstet Gynecol. 2017;217(4):471-2.

Cite this article as: Gupta T, Baruhee D, Agarwal S. A comparative study of the technical feasibility of complete salpingectomy versus tubal ligation as a method of tubal sterilisation during caesarean delivery. Int J Reprod Contracept Obstet Gynecol 2021;10:4482-7. 